\title{
Mathematical Modeling of Self-Oscillations in a Rijke's Tube with Variable Heat Flow Power
}

\author{
Boris Basok, Vladimir Gotsulenko
}

Department of Thermophysical Fundamentals of Energy-Saving Technologies, Institute of Engineering Thermal Physics of NAS of Ukraine, Kiev, Ukraine

Email address:

basok@ittf.kiev.ua (B. Basok), gosul@ukr.net (V. Gotsulenko)

\section{To cite this article:}

Boris Basok, Vladimir Gotsulenko. Mathematical Modeling of Self-Oscillations in a Rijke's Tube with Variable Heat Flow Power. American Journal of Mechanical and Industrial Engineering. Vol. 2, No. 1, 2017, pp. 48-53. doi: 10.11648/j.ajmie.20170201.18

Received: September 19, 2016; Accepted: September 29, 2016; Published: January 12, 2017

\begin{abstract}
In this paper the mathematical model of self - oscillation in Rijke's tube is found. We introduce the characteristic of the pressure of the heat supply. Using the energy equation in the form of the first law of thermodynamics to flow defined mechanisms of thermoacoustic instability in this problem. Using the pressure characteristic of the supply of heat and the classical Lyapunov's theory of stability defines the conditions for self-excitation of oscillation. It was found that when the increasing combustion delay the harmonic self-oscillations of the "singing" flame are converted to the relaxation oscillations.
\end{abstract}

Keywords: Rijke’s Tube, "Singing" Flame, Self-Oscillations, Combustion Delay Time, Instability, Head Characteristics of Heat Supply

\section{Introduction}

Small-amplitude thermoacoustic self-oscillations of oscillating (pulsating) combustion improve the economic and performance indices of combustion chambers of industrial units. However, as the thermal load increases, their amplitudes grow and become destructive. Serious complications and disturbances in operation are caused by pulsating combustion in blast-furnace stoves [1]. The results of investigations of this nonstationary regime by different authors are contradictory [2]. The same feature is also observed when the theoretical solutions [3] are compared to experimental investigations performed at a number of metallurgical plants.

The simplest device in which thermoacoustic oscillations are set up is the Rijke's tube. Its phenomenon lies in excitation of self-oscillations by heat supply from an internal heat source located in the lower part of a vertical tube that is open at both ends on formation of direct motion in the tube.

The mechanisms of thermoacoustic oscillations proposed by the author and considered below enable one to mathematically model the modes of periodic motions in the Rijke's tube. A complete qualitative coincidence of the results of the earlier experimental investigations and the theoretical solutions is observed.
As the air flow rate in the Rijke's tube increases, the temperature of the air moving in it decreases since the value $W$ of the heat flux is constant, which decreases its viscosity. This is responsible for the reduction in the hydraulic loss along the length with growth in the flow rate $Q$ in the laminar regime and for the formation of the descending branch $h_{\mathrm{fr}}(Q)$ of the negative hydraulic resistance. As the power $W$ decreases, the mechanism of negative hydro resistance is attenuated; the self-oscillations are maintained due to the delay $\tau$ of heat transfer at high-temperature gradients and volume relaxation of the heat supply [4]

Negative thermal resistance is responsible for the excitation of self-oscillations for a variable (flow-ratedependent) power $W$ of the heat flux. Apart from the phenomenological delay of the process of combustion, negative thermal resistance contributes to the formation of the "singing" flame mechanisms, which, under the corresponding conditions [4], can generate self-oscillations in the Rijke's tube when a burner is used for supply of heat instead of the electric coil.

Since the mechanisms of the Rijke's phenomenon of excitation of oscillations remained constant, theoretical descriptions of the Rijke's phenomenon rarely coincided with experimental ones even qualitatively [4]. Despite the seemingly contradictory experimental investigations of the 
Rijke's phenomenon, they are reliable and can be modeled mathematically but are very limited [5].

\section{Formulation of the Problem}

Motion in a vertical tube in which a "singing" flame occurs [4-5] may be natural (Rijke's tube) (Fig. 1) or be additionally created by a fan. The reason for the excitation of selfoscillations due to heat supply or their necessary condition is the presence of the ascending branch on the head characteristic of the Rijke's tube with an electric coil [5] and on the characteristic of heat supply of the singing flame. Furthermore, the mode and parameters of oscillations in the Rijke's tube are dependent, in a certain manner, on the phenomenological retardation of combustion $\tau$ and on the distinctive features of "turbulent" combustion where the electric coil is replaced by a gas burner burning gas.

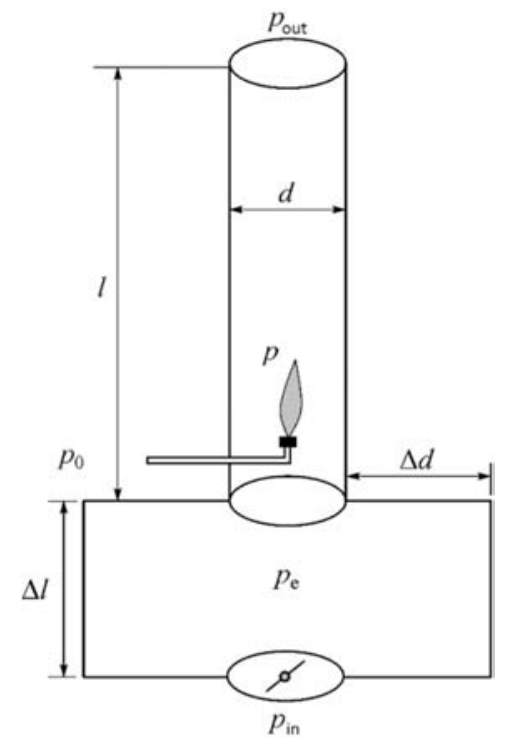

Fig. 1. Model of a Rijke's tube with a Singing'flame.

With decrease in the wave resistance $Z$ of the primary furnace, when the condition $Z<Z^{*}$ is fulfilled [6], thermoacoustic oscillations become relaxation ones with a constant amplitude and further reduction in $Z$ values. A regularity of such alternations in the flow parameters is growth in their amplitude with heat-supply power $W$, which is also observed in the furnaces of stoves of blast furnaces in which the dynamic load on the structure becomes destructive [5].

Since increase in the heat load and hence in the blowing temperature yields a multimillion economic effect only on one blast furnace due to the decrease in the burden coke, the problem of reduction in the oscillation amplitude of vibrational combustion is pressing. Below, we consider the solution of such a problem using mathematical modeling for self-oscillations of vibrational combustion by acting on the mechanisms of their sustaining and by possible changing the wave resistance of the oscillatory circuit. The efficiency of solution of this problem is illustrated by oscillations in a Rijke's tube (Fig. 1) when the supply of heat is from the combustion of fuel.

\section{Equations Describing Self-Oscillations in Rijke's Tube with Variable Heat Flow Power}

Apply the d'Alembert principle to the heated gas column after the combustion zone (Fig. 1), considering it as a material particle oscillating at self-oscillations as a single whole. Obtain

$$
m \frac{d w}{d t}=\left(p-p_{\text {out }}\right) S-m g-F_{\text {fr }},
$$

where $w$ is velocity of gas column motion, $m=\rho \ell S$ is mass of gas column, $S$ is area of normal section of the tube with diameter $d$ (Fig. 1), $F_{\mathrm{fr}}$ - the force of viscous friction on the side surface of the tube. Considering that: $p_{\text {out }}=p_{0}-\rho_{0} g \ell, h_{\mathrm{fr}}=\lambda \frac{\ell}{d} \rho \frac{w^{2}}{2}$, where $h_{\mathrm{fr}}=\frac{F_{\mathrm{fr}}}{S}, p_{0}$ is pressure in the ambient medium, $\lambda$ is coefficient of hydraulic losses, the equation of motion (1) will have the following final form:

$$
L_{\mathrm{a}} \frac{d Q}{d t}=F(Q)-P
$$

where: $L_{\mathrm{a}}=\frac{\rho \ell}{S}$ is acoustic mass of the heated gas column, $F(Q)=A(Q)-h_{\mathrm{fr}}(Q)-h_{\mathrm{T}}(Q)-$ head characteristics of heat supply [7-9], $A(Q)=g \ell\left(\rho_{0}-\rho\right)$ - lifting force pressure, $p$ - the pressure in the tube at the outlet of the combustion zone, $h_{\mathrm{T}}(Q)=p_{\mathrm{e}}-p-$ the hydraulic resistance of the combustion zone (thermal resistance) [9], $P=p_{0}-p_{\mathrm{e}}, h_{\mathrm{fr}}(Q)-$ dependence of viscous stress over the length of the heated part of the tube (Fig. 1) on volume flow rate of heated gas $Q=S w$.

Write the equation of mass conservation for gas in the volume located after the combustion zone (Fig. 1):

$$
d M(t)=\left(G_{\text {in }}(t-\tau)-G\right) d t,
$$

where: $M(t)$ is mass of cold gas in the volume in the moment of time $t, \tau$ - combustion time delay $G_{\text {in }}=\rho_{0} w_{0} S$ is gas mass flux incoming in the volume, and $G=\rho w S$ is mass-flow rate of heated gas. Note that

$$
d M=V_{\mathrm{e}} d \rho_{\mathrm{e}}, \frac{d p_{\mathrm{e}}}{d \rho_{\mathrm{e}}}=c_{\mathrm{e}}^{2}, c_{\mathrm{e}}=\sqrt{\gamma R T_{\mathrm{e}}},
$$

where $V_{\mathrm{e}}$ is tank volume in front of electric coil, $\rho_{\mathrm{e}}$ and $T_{\mathrm{e}}$ is density and temperature of gas in it, respectively, $c_{\mathrm{e}}$ is velocity of sound propagation in this volume, $\gamma$ is 
adiabatic exponent, and $R$ is gas constant. Thus, equation (3) may be written as follows:

$$
C_{\mathrm{a}} \frac{d p_{\mathrm{e}}}{d t}=\frac{\rho_{\mathrm{e}}}{\rho} Q_{0}-Q
$$

where $C_{\mathrm{a}}=\rho^{-1} c_{\mathrm{e}}^{-2} V_{\mathrm{e}}$ is acoustic flexibility of the volume in front of electric coil, and $Q=S w_{0}$ is volumetric flow rate of incoming cold gas.

To control gas flow rate through the vertical tube, the throttle is mounted at the receiver input (Fig. 1). From equation of hydraulic losses on this throttle it may be inferred that

$$
p_{\text {in }}-p_{\mathrm{e}}=k Q_{0}^{2},
$$

where $k$ is coefficient of hydraulic losses. Assuming $\Delta \ell<<\ell$ obtain that $p_{\text {in }} \approx p_{0}$ and according to (4), $\rho_{\mathrm{e}} \rho^{-1} Q_{0} \approx \eta \sqrt{p_{0}-p_{e}}, \eta=\rho_{\mathrm{e}}(\rho \sqrt{k})^{-1}$. Thus, equation (3) will be finally written as follows.

$$
C_{\mathrm{a}} \frac{d P}{d t}=Q-\varphi(P)
$$

assuming that $\varphi(P)=\eta \sqrt{P}$.

The obtained dynamic system (2), (5) formally coincides with equations of the theory of longitudinal self-oscillations (surging) in the compressor as a dynamic system with lumped parameters [15]. In the considered analogy the role of the introduced function $F(Q)$ is played by the head characteristic of the blower, determined experimentally. At that the head characteristic of the compressor characterizes part of mechanical energy of drive rotation, transformed in the head developed by the blower, and the introduced characteristic of heat supply $F(Q)$ determines part of heat, supplied to flow that is transformed into its head.

\section{Mechanisms of Thermoacoustic Oscillations for a Heat-Flux Power Varying with Flow Rate}

The presence of the descending branches on the $h_{\mathrm{fr}}(Q)$ or $h_{\mathrm{T}}(Q)$ plots and the growing character of change in the lift pressure $A(Q)$ contribute to the formation of the ascending branch of the head characteristic $F(Q)$.

To find the conditions of appearance of the $h_{\mathrm{T}}(Q)$ descending branch we take that the hydraulic head loss $h_{\mathrm{fr}}(Q)$ is absent, i.e., the compressible fluid is hydraulically ideal. Since the flow head decreases with growth in $h_{\mathrm{T}}(Q)$ and increases with its reduction, we determine the character of variation in the dependence $h_{\mathrm{T}}(Q)$ from the change in the head $F(Q)$ in heat supply. For this purpose we use the equation of the first law of thermodynamics in differential form for flow in a horizontal channel

$$
d q=d u+d\left(\frac{P}{\rho}\right)+d \frac{w^{2}}{2}
$$

or

$$
d F(Q)=\rho d(q-u)
$$

Since the heat supplied in the polytropic process is $d q=c_{\mathrm{v}} \frac{n-k}{n-1} d T$, and the change in the internal energy is $d u=c_{\mathrm{v}} d T$, with allowance for the dependence of the temperature on the volumetric flow rate $T=T_{0}+\psi(Q)$ we may write Eq. (7) as

$$
d F(Q)=\rho c_{\mathrm{v}} \frac{1-k}{n-1} d \psi(Q) .
$$

If the function $\psi(Q)$ is increasing, the ascending branch of the function $F(Q)$ results from the descending branch of $h_{\mathrm{T}}(Q)$ and, according to Eq. (8), occurs in the polytropic process of heat supply with an exponent of the process $n$ for which $0 \leq n<1$, which is realized in vertical primary furnaces.

In the case where the function $\psi(Q)$ is decreasing, the fulfillment of the inequality $n>1$ is required for the descending branch to occur on the $h_{\mathrm{T}}(Q)$ plot. Thus, under the conditions given above, the ascending branch of the characteristic $F(Q)$ in the nonviscous-gas flow appears because of the descending branch of $h_{\mathrm{T}}(Q)$. An analogous result is obtained in analyzing the head loss equal to the difference of the total pressures and caused by the heat supply in the flow of an ideal gas.

The lift pressure $A(Q)$ is involved in the head characteristic $F(Q)$ and contributes to the generation of its ascending branch, if the function $\psi(Q)$ in the device (Fig. 1) increases. This is the second reason why self-oscillations are excited in vertical primary furnaces; this reason is the most substantial for blast-furnace stoves.

The increase in the hydraulic resistance of the throttle in the device (Fig. 1) contributes to the fact that the temperature in the flow will grow with heat load more appreciably than velocity. Under this condition, the Reynolds number decreases with increase in the flow rate. The $h_{\mathrm{fr}}(Q)$ descending branch of hydraulic loss along the length is formed in the process of turbulent-to-laminar transition because of the reduction in the loss, which is the third mechanism of thermoacoustic oscillations of a "singing" flame. Further increase in the flow rate will contribute to the growth in the hydraulic loss in accordance with the laminar regime of motion, if it is preserved. 


\section{Conditions of Excitation of Vibrational Combustion}

Self-excitation of self-oscillations in Rijke's tube results from the loss of stability of stationary convective flow of heated gas that correlates with the positioning of the dynamic system equilibrium (2), (5). The character of equilibrium position stability is determined by the characters of real components of respective Lyapunov characteristic indicators [10]. In the considered problem they take the form:

$$
\lambda_{1,2}=\frac{\alpha}{L_{\mathrm{a}}}-\frac{\beta}{C_{\mathrm{a}}} \pm \sqrt{\left(\frac{\alpha}{L_{\mathrm{a}}}+\frac{\beta}{C_{\mathrm{a}}}\right)^{2}-\frac{4}{L_{\mathrm{a}} C_{\mathrm{a}}}},
$$

where $\quad \alpha=\left.\frac{d F(Q)}{d Q}\right|_{Q=\xi}, \quad \beta=\left.\frac{d \varphi(P)}{d P}\right|_{P=F(\xi)}, \quad \xi-$ is stationary volume flow rate of heated gas determined from the equation $F(\xi)=\left(\frac{\xi}{\eta}\right)^{2}$. It can be easily verified that

$$
\operatorname{Re}\left\{\lambda_{1,2}\right\}>0 \Leftrightarrow \alpha>\left.0 \Leftrightarrow \frac{d F}{d Q}\right|_{Q=\xi}>0
$$

From correlation (9) it may be inferred that at the performance of inequality $\left.\frac{d F(Q)}{d Q}\right|_{Q=\xi}<0$ the stationary mode of the flow determined by the flow rate $Q=\xi$ is stable, and in this case self-excitation of self-oscillations is impossible. Thus, for self-excitation of self-oscillations there

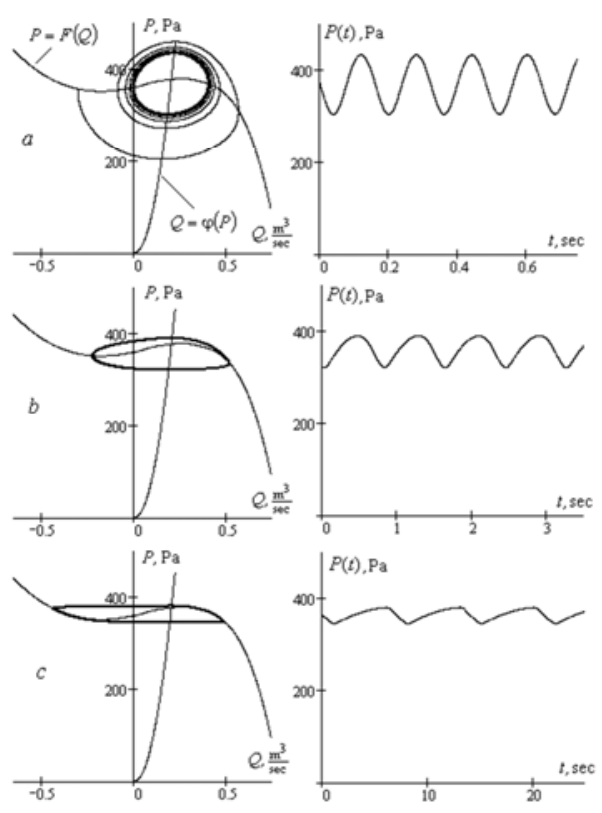

is a need of the zone of "negative" strength, where with an increase of the flow rate $Q$ the head $F$ of the heated gas column does not decrease but vice versa increases, i.e. the inequality $\frac{d F(Q)}{d Q}>0$ is performed.

The condition (10) is necessary for self-excitation of selfoscillations. The sufficient condition of their appearance is existence of the limit cycle in the equation of integral curves [9]

$$
C_{\mathrm{a}}\{F(Q)-P\} d P=L_{\mathrm{a}}\{Q-\varphi(P)\} d Q .
$$

Dimensions and form of the limit cycle of equation (11) are determined by the values of acoustic parameters $L_{\mathrm{a}}$ and $C_{\mathrm{a}}$.

\section{The Results of Mathematical Modeling}

Self-oscillations are harmonic, when the values of the wave resistance $Z=\sqrt{\frac{L_{\mathrm{a}}}{C_{\mathrm{a}}}}$ are high. Their amplitudes increase with manifestation of the action of phenomenological retardation (Fig. 2). As the wave resistance decreases, the oscillations become relaxation ones, whereas under the condition that $Z<Z^{*}$ their amplitudes are independent of further decrease in the quantity $Z$ and on the change in the flow rate $Q$ in the system, as has already been noted. Upon the transition to relaxation oscillations, the dependence of the amplitude on the manifestation of the retardation $\tau$ becomes weaker.

The limit cycle of constant-amplitude relaxation selfoscillations or the cycle close to it (Fig. 2) is independent of the retardation $\tau$.

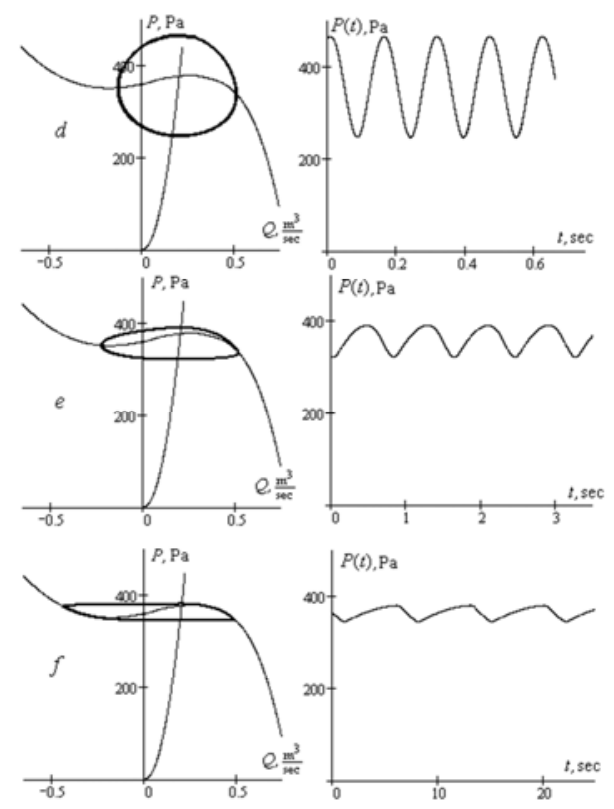

Fig. 2. Transformation of the limit cycle of harmonic oscillations to a limit cycle of relaxation oscillations of variable and constant amplitudes with decrease in the wave resistance: $a$ and d) $Z=325.09, b$ and e) 79.63 , and $c$ and f) $\left.17.8 \frac{\mathrm{Pa} \cdot \mathrm{sec}}{\mathrm{m}^{3}} ; a-c\right) \tau=0$ and $\left.d-f\right) 0.04 \mathrm{sec}$ 
Thus, for the vibrational-combustion mechanism due to the phenomenological retardation, the oscillation amplitude remains constant under the conditions in question. In such a regime, self -oscillations are mainly determined by the character of heat-to-head conversion, i.e., by the head characteristic $F(Q)$. The limit cycle increases with heatflux power [7, 9].

An efficient method for controlling thermoacoustic selfoscillations of a "singing" flame is the introduction of the active vortex resistance $h_{\mathrm{wh}}=k_{\mathrm{wh}} Q^{2}$, in which $k_{\text {wh }}=$ var , into the primary furnace. In this case we have a controlled change in the characteristic $F(Q)$, which enables us to control self-oscillations [7]. The same limit cycle of constant-amplitude oscillations is preserved in the case of series connection of a passive oscillatory circuit of dynamic damping, through which straight-through motion is carried out.

The second efficient method for controlling and neutralizing constant-amplitude oscillations is the transformation of the primary furnace to a "honeycomb" one (Fig. 3), in which the acoustic masses $L_{\mathrm{a}_{\mathrm{i}}}$ of each individual primary furnace are increased, and the acoustic flexibility of oscillatory circuits may be variable. In this structure, a constant limit cycle becomes variable due to the increase in the wave resistance $Z=\sqrt{\frac{L_{\mathrm{a}_{\mathrm{i}}}}{C_{\mathrm{a}, \mathrm{var}}}}$ in individual primary furnaces [7], and it is brought closer to the cycle of nearly harmonic oscillations by changing the quantity $C_{\mathrm{a} \text {,var }}$, if it seems possible for the corresponding structure.

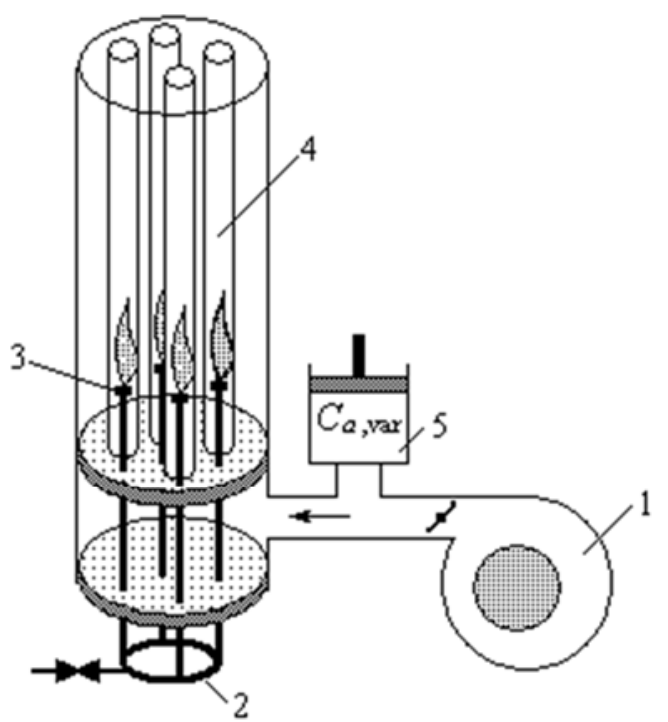

Fig. 3. Honeycomb"primary furnace with individual burners and a variable acoustic flexibility $C_{\mathrm{a}, \mathrm{var}}$ : 1) fan; 2) gas collector; 3) atomizers; 4) honeycomb primary furnaces; 5) additional mass accumulator of the oscillatory circuit.

The character of motions in devices of a "honeycomb" primary furnace corresponds to limit cycles dependent on the wave resistance $Z$ and determined by the condition $Z<Z^{*}$ [6] (Fig. 2), whereas the harmonic oscillations are easily controlled.

\section{Conclusions}

When the harmonic oscillations of vibrational combustion are transformed to relaxation ones, the influence of the phenomenological combustion retardation on the oscillation amplitude becomes weaker. The formation of a limit cycle of constant-amplitude relaxation oscillations or of that close to it totally eliminates such influence and the value of the amplitude for these conditions is mainly determined by the intensity of conversion of heat to the flow head, growing with heat-supply power.

Relaxation self-oscillations corresponding to the limit cycle of constant-amplitude oscillations do not change the value of the amplitude in dynamic damping.

Control of such oscillations is difficult; it involves the action on the mechanisms of their excitation and sustaining by the use of which one can decrease the values of the intensity $\left|\frac{d F(Q)}{d Q}\right|$ of the ascending branch of the head characteristic $F(Q)$.

Increase in the wave resistance $Z$ of the oscillatory circuit transforms the limit cycle of constant-amplitude oscillations to a variable cycle, which enables one to control the oscillations; a certain influence of the mechanisms of phenomenological retardation on the oscillation amplitude manifests itself.

\section{References}

[1] Larinov V. M., Zaripov R. G. Self-Oscillations of the Gas in Combustion-Involving Plants [in Russian]. Kazan: Izd. Kazansk. Gos. Tekhn. Univ. 2003. 327 pp.

[2] Shklyar F. R., Malkin V. M., Kashtanova S. P., Kalugin Ya. P., and Sovetkin V. L. Blast Furnace Stoves [in Russian]. Moscow: Metallurgiya. 1982. 176 pp.

[3] Zuker D., Glas P., and Beneke G. "Pressure pulsations in air heaters // Chern. Metally. - 1980. - No. 22. P. 20-26.

[4] Belyaev N. M., Belik N. P., and Pol'shin A. V. Thermoacoustic Oscillations of Gas-Liquid Flows in Complex Pipeline Power Plants [in Russian]. Kiev-Donetsk: Vysshaya Shkola, 1985. $160 \mathrm{pp}$.

[5] Larinov V. M., Zaripov R. G. Self-Oscillations of the Gas in Combustion-Involving Plants [in Russian]. Kazan: Izd. Kazansk. Gos. Tekhn. Univ. 2003. 327 pp.

[6] V. V. Gotsulenko. Special modes of the Rijke's phenomenon// Journal of Engineering Physics and Thermophysics. - 2007. Vol. 78, No. 2. P. 375-379.

[7] Gotsulenko V. V On the problem of control of relaxation oscillations of a "singing" flame // Journal of Engineering Physics and Thermophysics. - 2007. - Vol. 80, No. 3. P. 563569. 
[8] Basok B. I. Gotsulenko V. V. Self-oscillations in a Rijke's tube with receiver positioning at its input // Thermophysics and Aeromechanics. - 2014. - Vol. 21, No. 4. - P. 487-496.

[9] Basok B. I. Gotsulenko V.V. Calculating the Parameters of Self - Oscillations in the Vertical Combustion Chamber of the

\section{Biography}

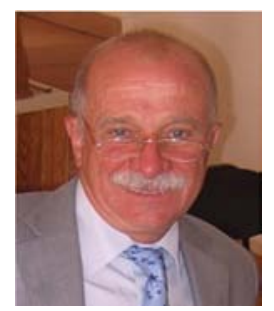

Boris Ivanovich Basok, doctor of technical Sciences, Professor, corresponding member of NAS of Ukraine, Deputy Director of the Institute of Engineering Thermal Physics of NAS of Ukraine.
Blast - Furnace Air Heater during Unstable Combustion // Thermal Engineering. - 2015, Vol. 62, No. 1. - P. 58-63.

[10] Demidovich B. P. Lectures on the mathematical theory of stability [in Russian]. Moscow: Nauka, 1967. 472 pp.

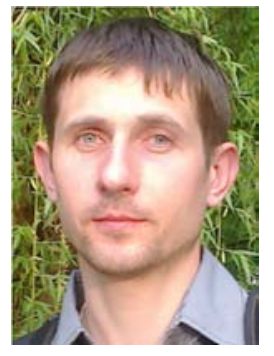

Vladimir Vladimirovich Gotsulenko, doctor of technical Sciences, senior researcher of the Department of thermophysical fundamentals of energy-saving technologies of the Institute of Engineering Thermal Physics of NAS of Ukraine.

Institute of Engineering Thermal Physics of NAS of Ukraine. Kiev 03057, Zhelyabova str., $2 \mathrm{~A}$. 